\title{
LA STAZIONE SISMICA DI MESSINA
}

\author{
Antonino Girlanda
}

Uno degli scopi principali per cui fu istituito, verso il 1876 , l'Osservatorio di Messina fu quello delle osservazioni sismiche. L'Osservatorio, che in un primo tempo fu aggregato all'Istituto Nautico, ebbe inizialmente soltanto la possibilità di eseguire la determinazione astronomica del tempo, le ordinarie osservazioni meteorologiche e la regolazione dei cronometri delle numerose navi che toccavano il porto di Messina.

Nel 1896 il Comune di Messina, riconosciuta l'insufficienza e l'irrazionale ubicazione del locale fino allora destinato ad osservatorio, intraprese la costruzione di un nuovo edifizio sulla collina dell'Andria, sorgente alla periferia della città, in località allora riconosciuta come la più idonea alle osservazioni geofisiche in genere, e a quelle sismiche in particolare. La costruzione ebbe termine nel 1902 e, l'anno successivo, il Consiglio Provinciale e il Comune di Messina, su proposta della Facoltà di Scienze, deliberarono di farne la consegna all'Università e di affidare la direzione del nuovo Osservatorio al professore titolare di fisica terrestre, la cui cattedra era in corso di istituzione. Nel 1904 l'Osservatorio di Messina divenne così l'Istituto di Fisica Terrestre e di Meteorologia dell'Università.

Gli strumenti destinati alle prime osservazioni sismiche furono alcuni "sismoscopi avvisatori" di vario tipo e un sismografo a due componenti, costruito su un modello di Agamennone. Questo sismografo, che con i sismoscopi era stato fornito dall'Ufficio di Meteorologia e Geodinamica, era costituito da un pendolo della lunghezza di 15 metri, con una massa di $300 \mathrm{~kg}$; la carta si svolgeva con una velocità di circa 40 centimetri all'ora e il tempo veniva segnato ogni mezz'ora.

Sin d'allora si era ravvisata la necessità di attrezzare l'Istituto di strumenti più sensibili e completi $e$, in seguito al grave terremoto, che l'8 settembre 1905 colpì duramente la Calabria e parte della Sicilia, venne accelerato l'impianto di un microsismografo Vicentini, che iniziò il suo regolare funzionamento il $1^{\circ}$ gennaio 1906.

Verso la fine del 1908 era in via di realizzazione un notevole in- 


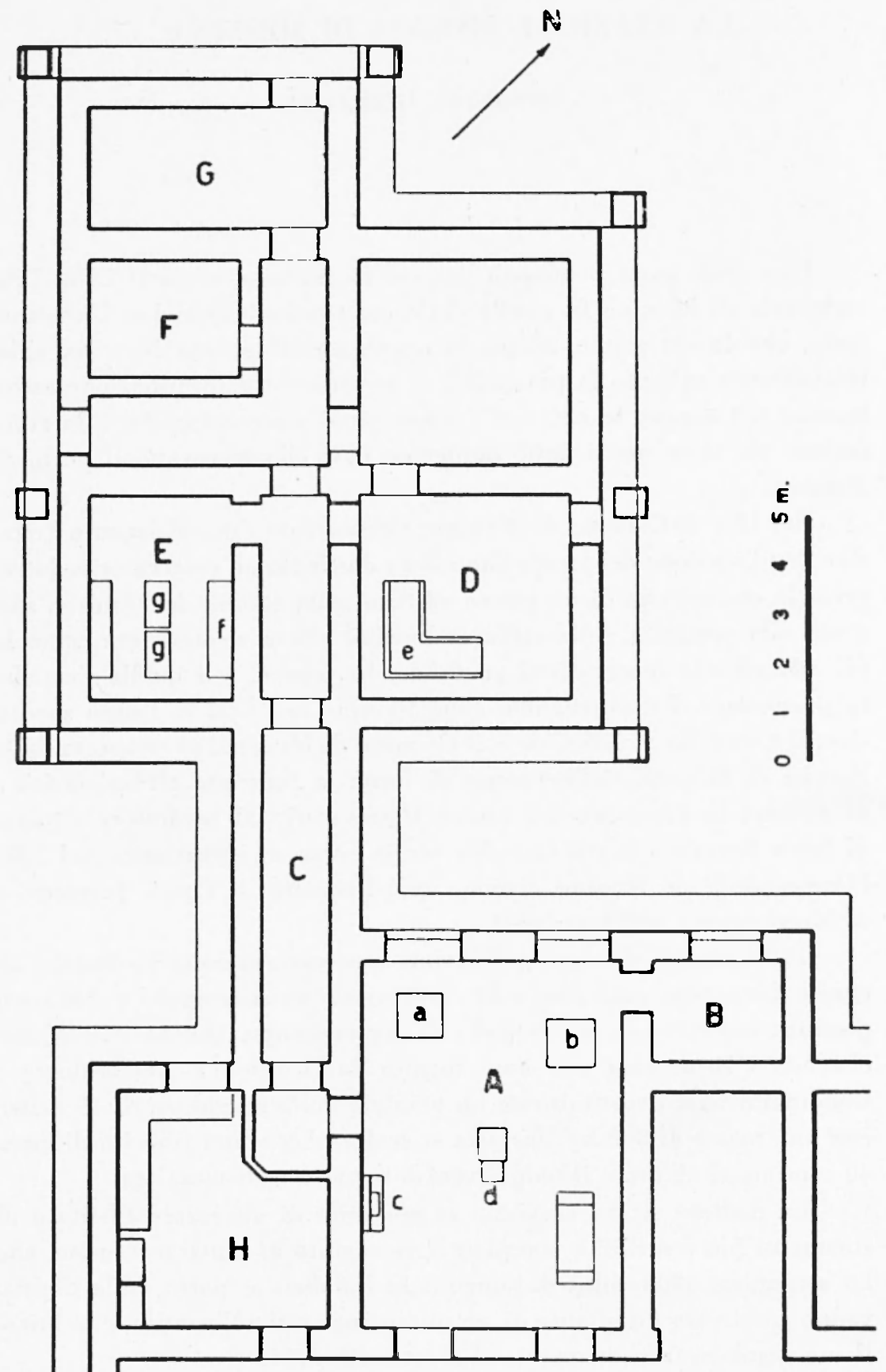

Fig. 1 
cremento dell'attrezzatura sismica con l'impianto di due pendoli orizzontali del Mainka, costruiti a Messina, e di un Wiechert orizzontale da $200 \mathrm{~kg}$, acquistato a Göttingen da Splindler e Hoyer. Il grande terremoto, che all'alba del 28 dicembre di quell'anno distrusse quasi totalmente Messina, paralizzò tutte le attività: i due Mainka furono distruti nel crollo di un'ala dell'edifizio e il Vicentini gravemente dan-

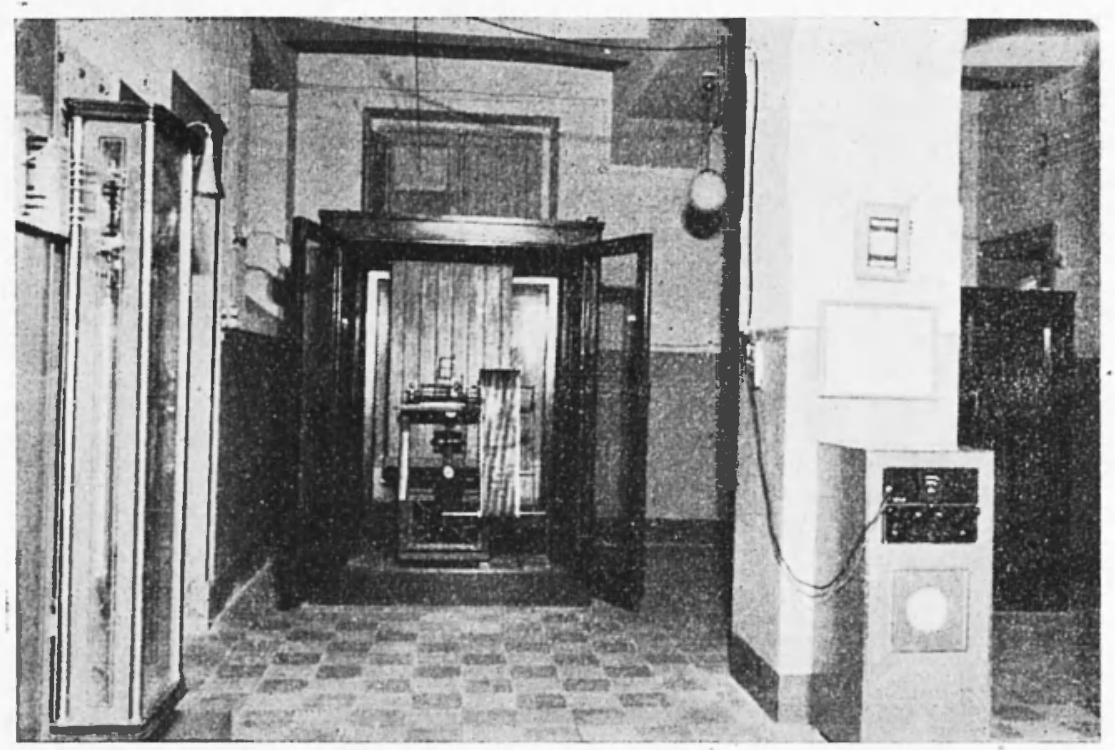

Fig. 2

neggriato. Il disastro che commosse l'opinione pubblica mondiale, desto, come arviene greneralmente in simili occasioni, un rrande interesse nei confronti dei problemi riguardanti la sismologia e numerosi furono 1 programmi e le proposte per l'organizzazione di un servizio sismico cflicicnte. Cosi, la Commissione Reale, incaricata di designare le aree più adatte per la ricostruzione dei fabbricati, nel presentare il rendiconto dei lavori più urgenti da essa eseguiti nel $1^{\circ}$ semestre del 1909. avera formulato una serie di proposte complementari tra le quali figurava la seguente: che in Calabria sorgessero delle stazioni sismiche secondarie funzionanti sotto la direzione dell'Osservatorio di Messina. Cosi, durante la terza riunione della Commissione permanente della Associazione Sismologica Internazionale, tenutasi a Zermatt nel 1909, il delegalo del Governo italiano, prof. Luigi Palazzo, avanzó la propo- 
sta di istituire, fra gli osservatori di Messina, Catania e gli osservatori in Calabria, un servizio di " triangolazione sismica, con collegamento telegrafico, per lo studio delle velocita di propagazione delle onde sismiche, conformemente a quanto si era gia fatto in Giappone e conformemente alle idee che in quel tempo andavano sviluppandosi per opera di Kövesligrety. Le vicende parlamentari del tempo e il susseguirsi di difficolta di vario genere non permisero, né allora né in seguito, l'attuazione di questi brillanti disegni.

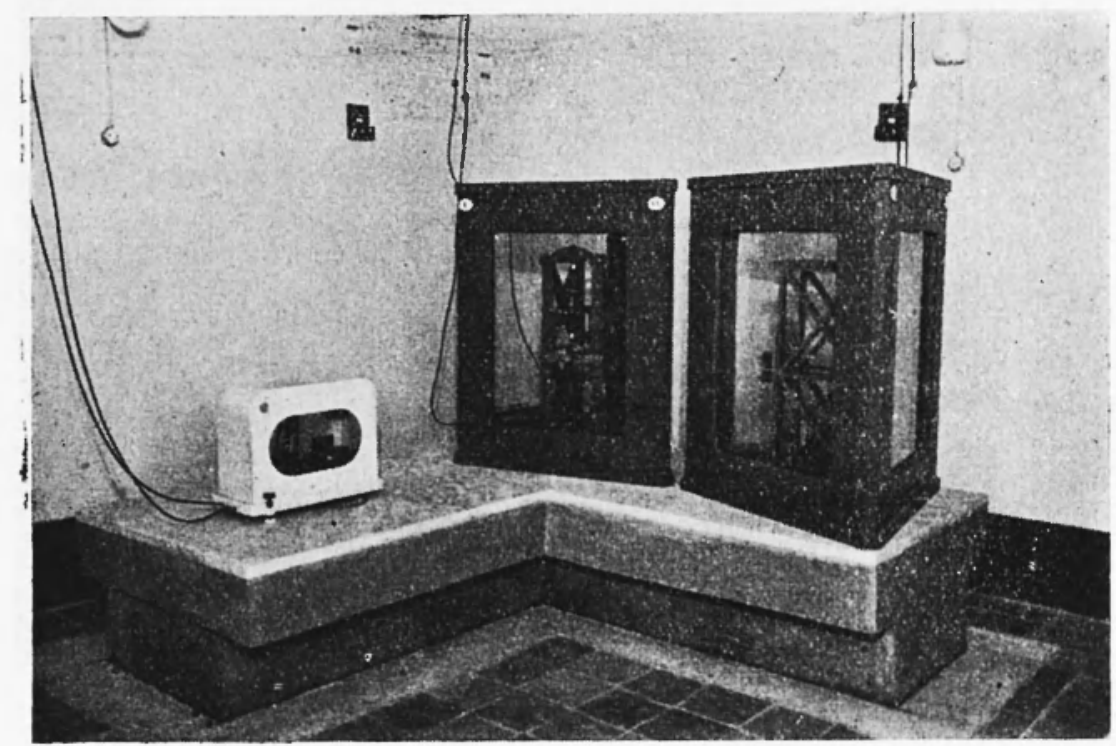

Fig. 3

Le osservazioni sismiche furono continuate con l'ausilio del sismografo Vicentini, rapidamente riparato e collocato nel sotterraneo dell'Istituto, che era rimasto quasi illeso. Nel 1912 l'Istituto fu dotato di un pendolo orizzontale Conrad e di due pendoli orizzontali di Alfani a registrazione meccanica. Questa attrezzatura non $f u$ sostanzialmente modificata fino al 1947, anno in cui l'Istituto Nazionale di Geofisica, ravvisando la necessità di disporre in Messina di un osservatorio facente parte della rete geofisica italiana, stipulava con l'Universita una convenzione in seguito alla quale veniva impiantata la nuova stazione sismica di Messina.

In un primo tempo fu impiantato un sismografo verticale di tipo Wiechert avente la massa di $80 \mathrm{~kg}$, costruito nelle officine dell'I.N.G. 


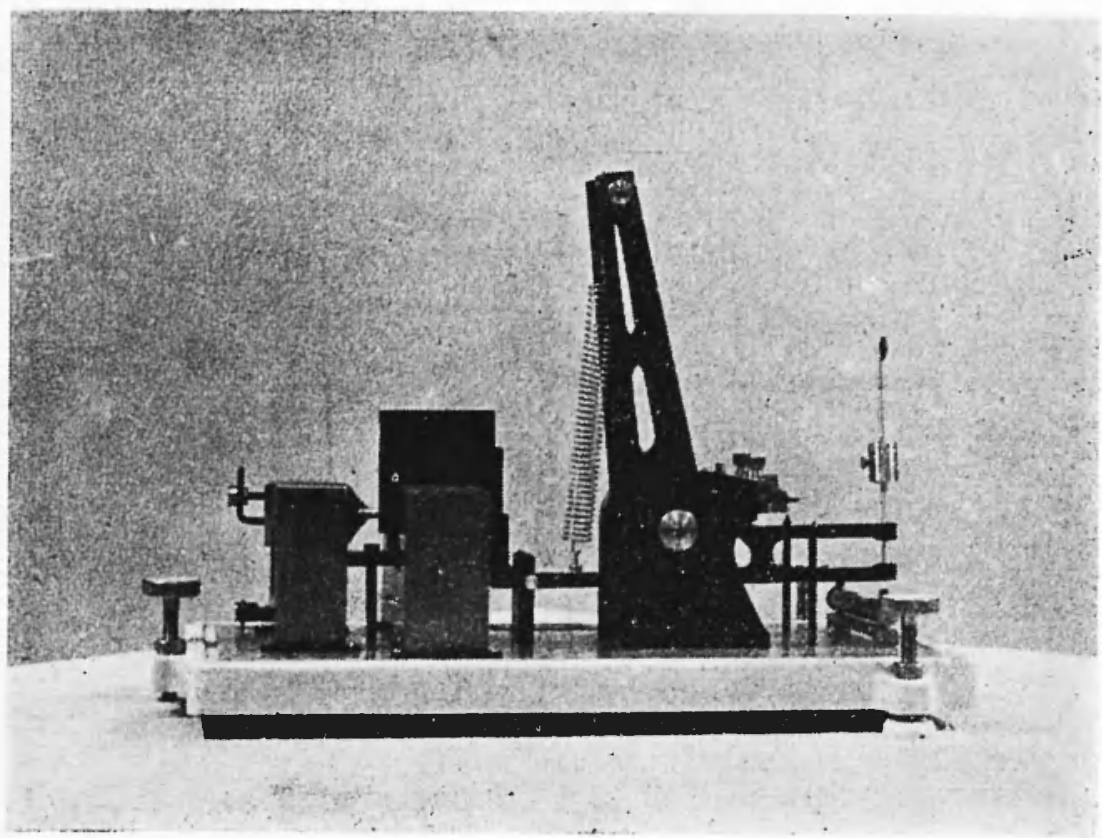

Fig. 4

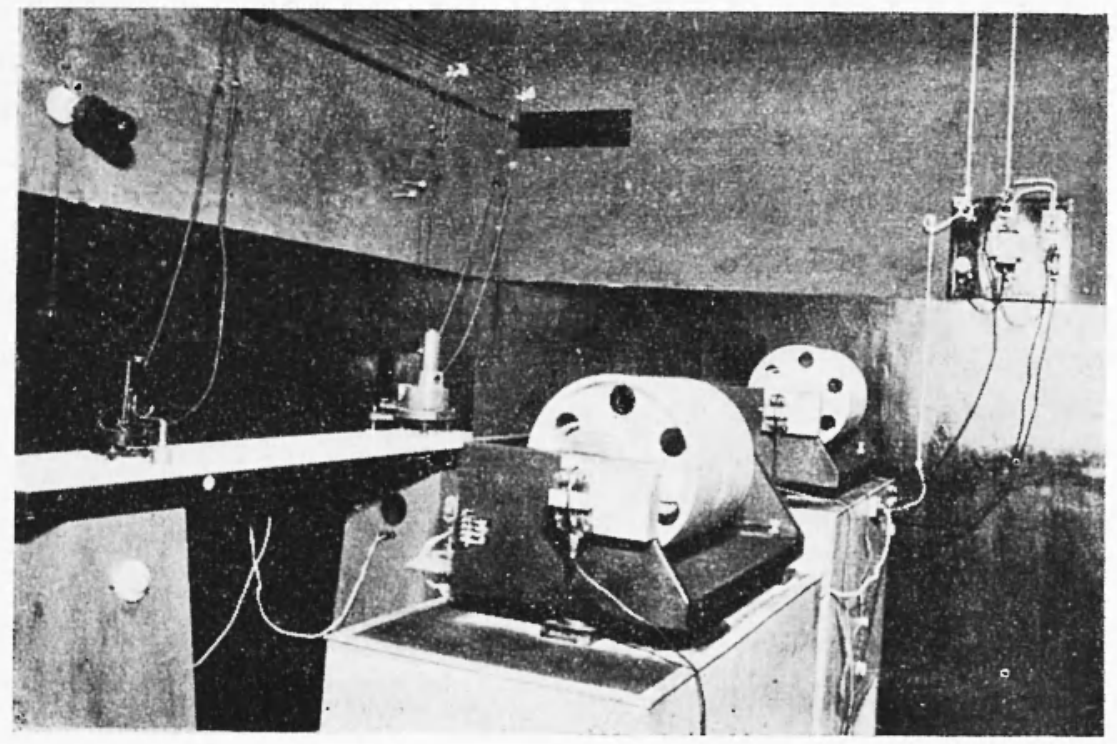

Fig. 5 
e dotato del nuovo tipo di smorzatore ad aria adottato dall'Istituto Nazionale di Geofisica per i sismografi dello stesso tipo funzionanti negli osservatori della rete sismica italiana ( $\left.{ }^{1}\right)$; fu, inoltre, messo in efficicnza

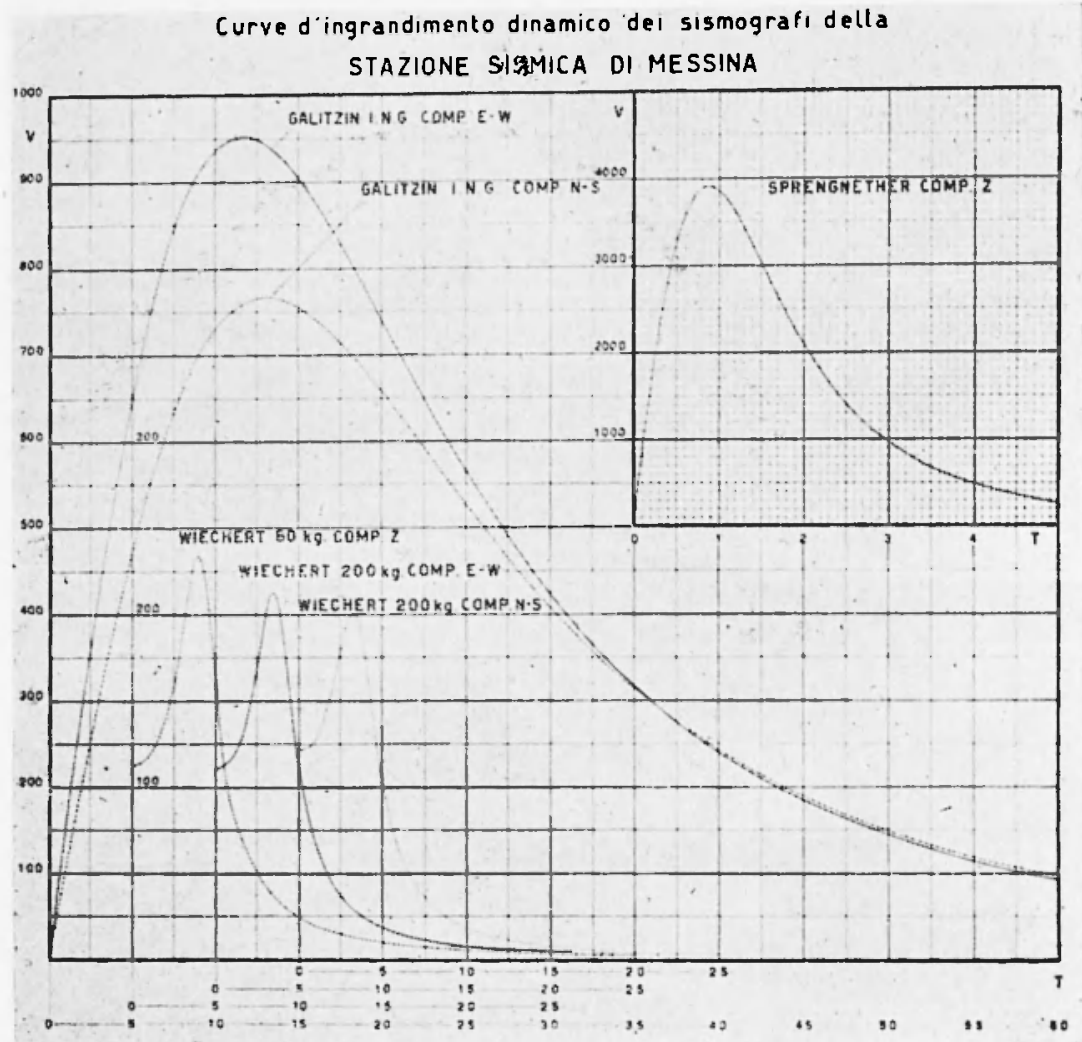

Fig. 6

il vecchio sismografo orizzontale Wiechert con la sostituzione completa del meccanismo di registrazione e smorzamento.

In seguito l'Istituto Geofisico ebbe assegnato, sui fondi del piano E.R.P., un sismografo verticale Sprengnether a breve periodo, corredato di un galvanometro e di due registratori folografici. Tale sismografo, com'è noto, è essenzialmente costituito da un pendolo verticale di tipo Galitzin. L'asse di rotazione è determinato da due coppie di laminette d'acciaio incrociate e la massa del sistema oscillante è distribuita in modo che la molla di sospensione sopporti il peso di poche diecine di grammi; qucsta circostanza permette l'uso di una molla di 


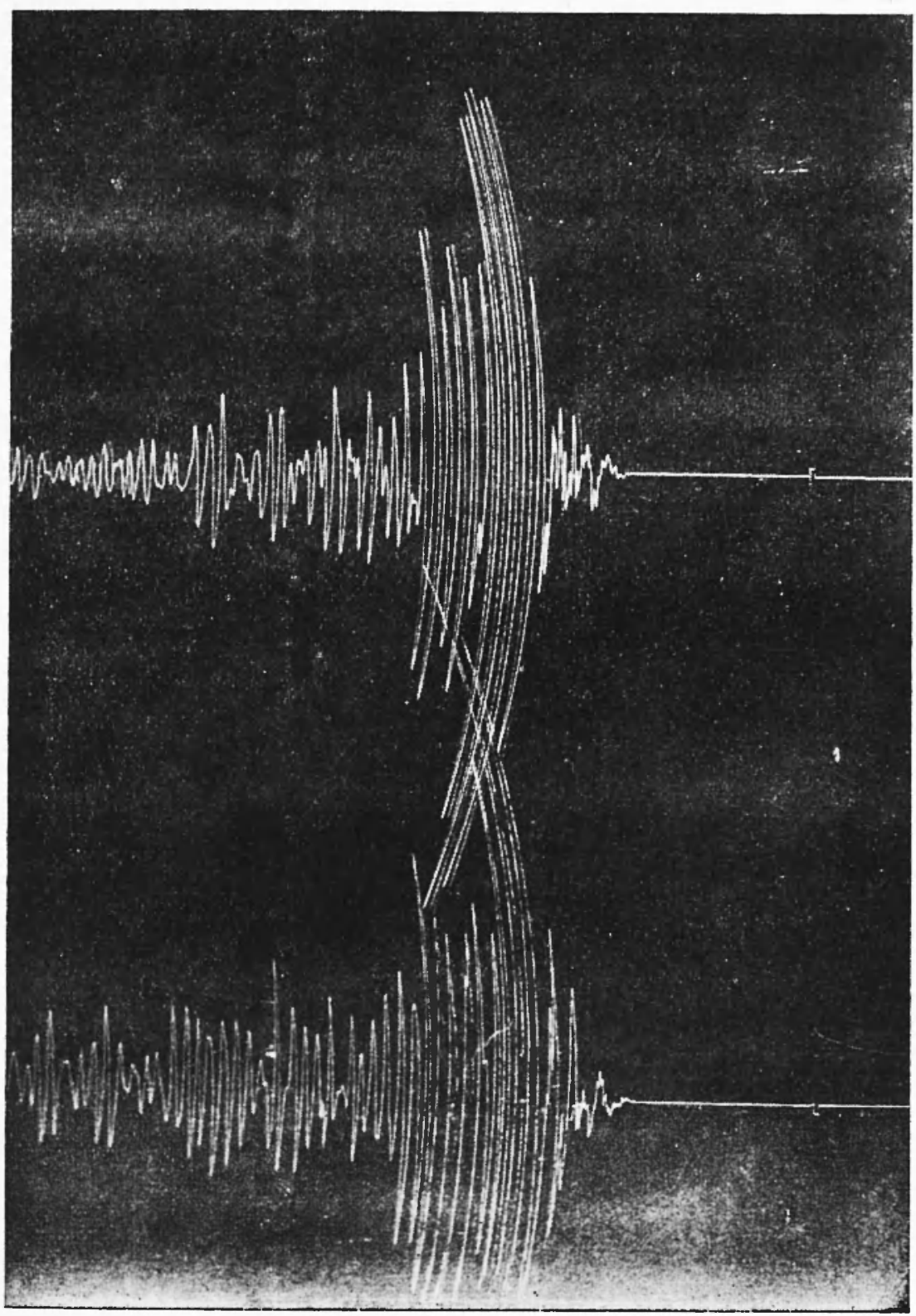

Fig. 7 

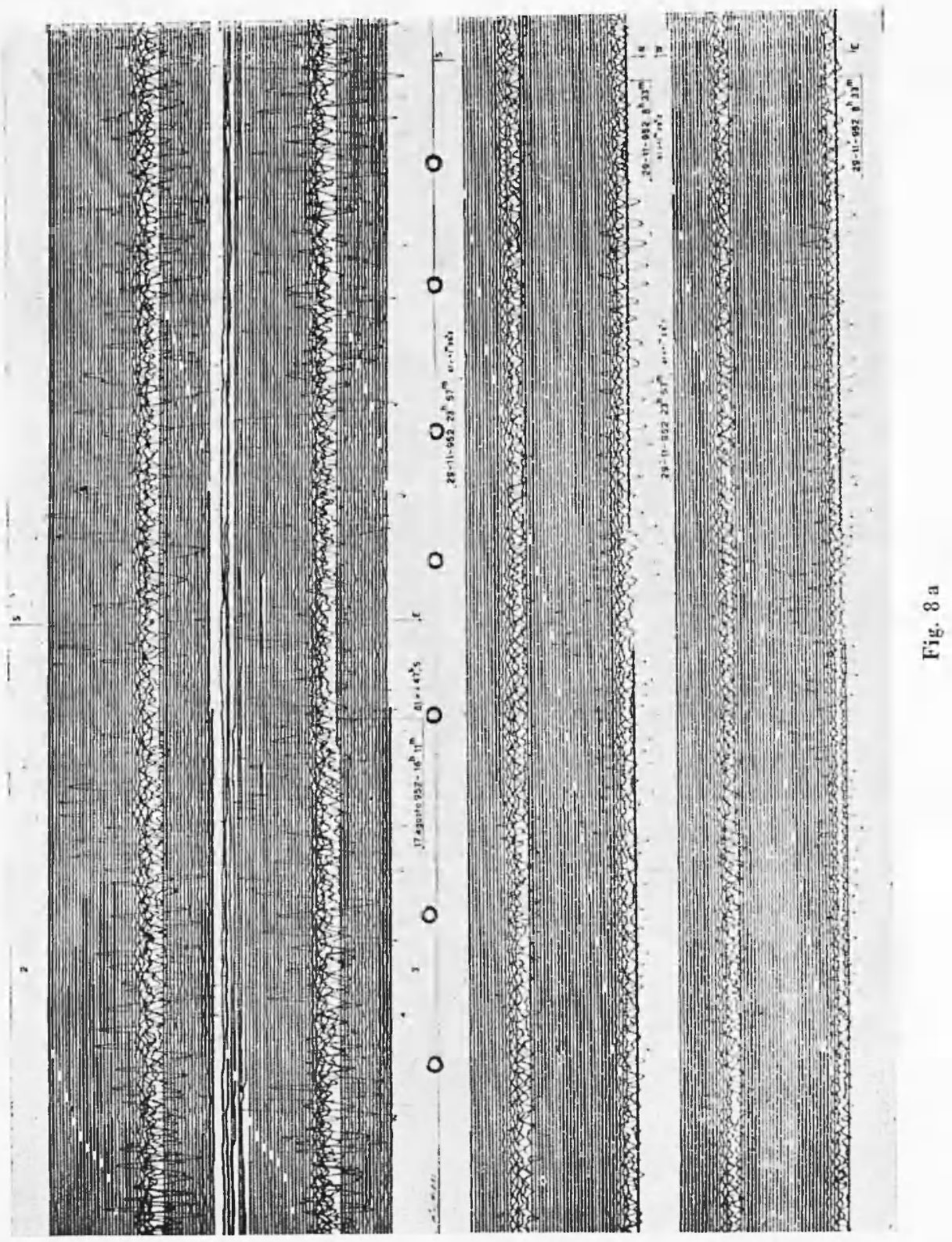
sospensione relativamente debole e, conseguentemente rende rapida ed agevole la regolazione e minimo l'effetto di temperatura sulle costanti elastiche della molla stessa. La bobina oscilla nel campo magnetico, non regolabile, generato da un magnete permanente ad elevata magnetizzazione, fissato alla base dello strumento. L'amplificazione, qualora se ne ravvisi la necessità, può essere ridotta per mezzo di un opportuno shunt magnetico. Lo smorzamento è ottenuto per mezzo di un'aletta di rame oscillante nel campo magnetico regolabile di un secondo magnete anch'esso fissalo alla base.

L'Istituto Nazionale di Geofisica ha completato, infine, la attrezzatura strumentale con l'invio di due sismografi orizzontali Galitzin del tipo I.N.G. Per avere la possibilità di impiegare i due registratori Sprengnether, non si è potuto adottare il sistema speciale di registrazione introdotto nella stazione sismica di Roma ( $\left.{ }^{2}\right)$ che permette il funzionamento dei registratori nello stesso ambiente in cui sono collocati i sismografi. Si è dovuto, pertanto, allestire un locale, adiacente alla sala dei sismografi, per adibirlo a sala di registrazione. Per registrare le due componenti orizzontali Galitzin su un unico foglio, è stato modificato uno dei due registratori fotografici Sprengnether, dimezzando Ia velocita di rotazione del tamburo e munendolo di un secondo riflettore.

Le coordinate geograficlıe della stazione sono le seguenti:

$$
\begin{aligned}
& \text { latitudine }=38^{\circ} \quad 11^{\prime}, 8 \quad \mathrm{~N}, \\
& \text { longritudine }=15^{\prime \prime} \quad 33^{\prime}, 3 \quad \mathrm{E}, \\
& \text { altitudine: m. } 15 \text { sul livello del mare. }
\end{aligned}
$$

Il terreno su cui porgria la stazione é costituito da alluvione rossa quatcrnaria a facies sabbiosa, ghiaiosa, leggermente argillosa; l'invasamento i costituito da uno zoccolo scistoso cristallino.

I locali destinati alle osservazioni sismiche e ai servizi, come si vede dalla planimetria riportata nella fig. 1 , sono così distribuiti :

A - Sala dei sismografi a regristrazione meccanica, nella quale in $a$ (fig. 2 ) è collocato il sismografo Wicchert verticale, in $b$ il sismografo Wiechert orizzontale, in $c$ il pendolo regrolatore Riefler per i segnali del tempo, in $d$ un apparecchio radio per la recezione dei segnali orari;

B - Saletta per geli accumulatori;

C - Corridoio di accesso ai locali destinati ai sismografi ad amplificazione elettromagnetica;

D - Sala dei sismografi ad amplificazione elettromagnetica, nella 


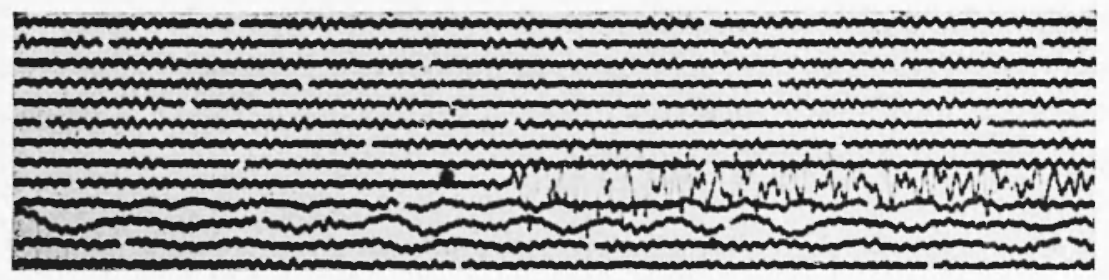

Fì. 8 b

quale in e, su un unico basamento, sono collocati i due sismografi Galitzin (fig. 3) e il sismografo Sprengnether (fig. 4);

E - Saletta per la registrazione fotografica, nella quale in $f$, su apposita mensola, sono collocati i galvanometri e in $g$, su appositi supporti, i due registratori fotografici (fig. 5);

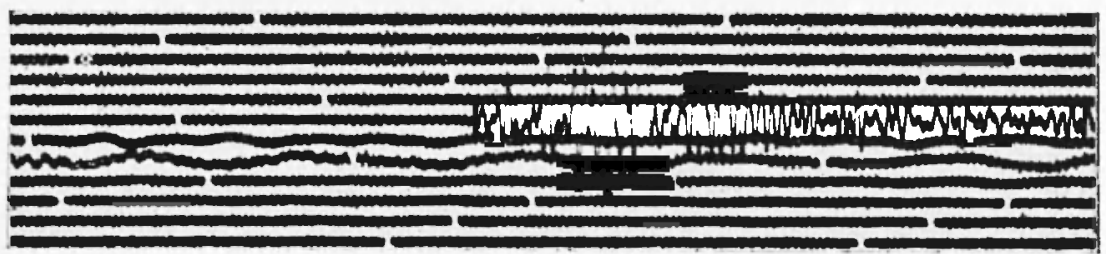

Fig. $8 \mathrm{c}$

F - Camera oscura per la preparazione delle carte dei sismografi a registrazione fotografica;

G - Saletta dei clinometri il cui impianto è di prossima realizzazione;

H - Camera oscura per lo sviluppo e il fissaggio delle carte fotografiche.

I valori delle costanti strumentali, quali risultano nella ultima determinazione eseguita nell'agosto 1952, sono riportati nelle tabelle seguenti :

\section{Compo-}

$\begin{array}{lllllllll}\text { nente } & T_{i r} & T s & V m & K & u & A & l & \text { I) }\end{array}$

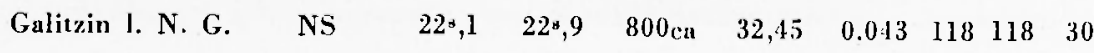

$\begin{array}{llllllllll}\text { Galizzin 1. N. G. } & \text { EW } & 20 * 7 & 19 \times, 7 & 952 \times a & 49,70 & 0,016 & 105 & 115 & 30\end{array}$

$\begin{array}{cccccccc} & \begin{array}{c}\text { Compo- } \\ \text { nente }\end{array} & T_{g} & T_{s} & V_{m} & u & k+1 & \text { I) } \\ \text { Sprengnether } & V & 14,5 & 18,5 & 3.900 & 0.000 & 8.000 & 60\end{array}$




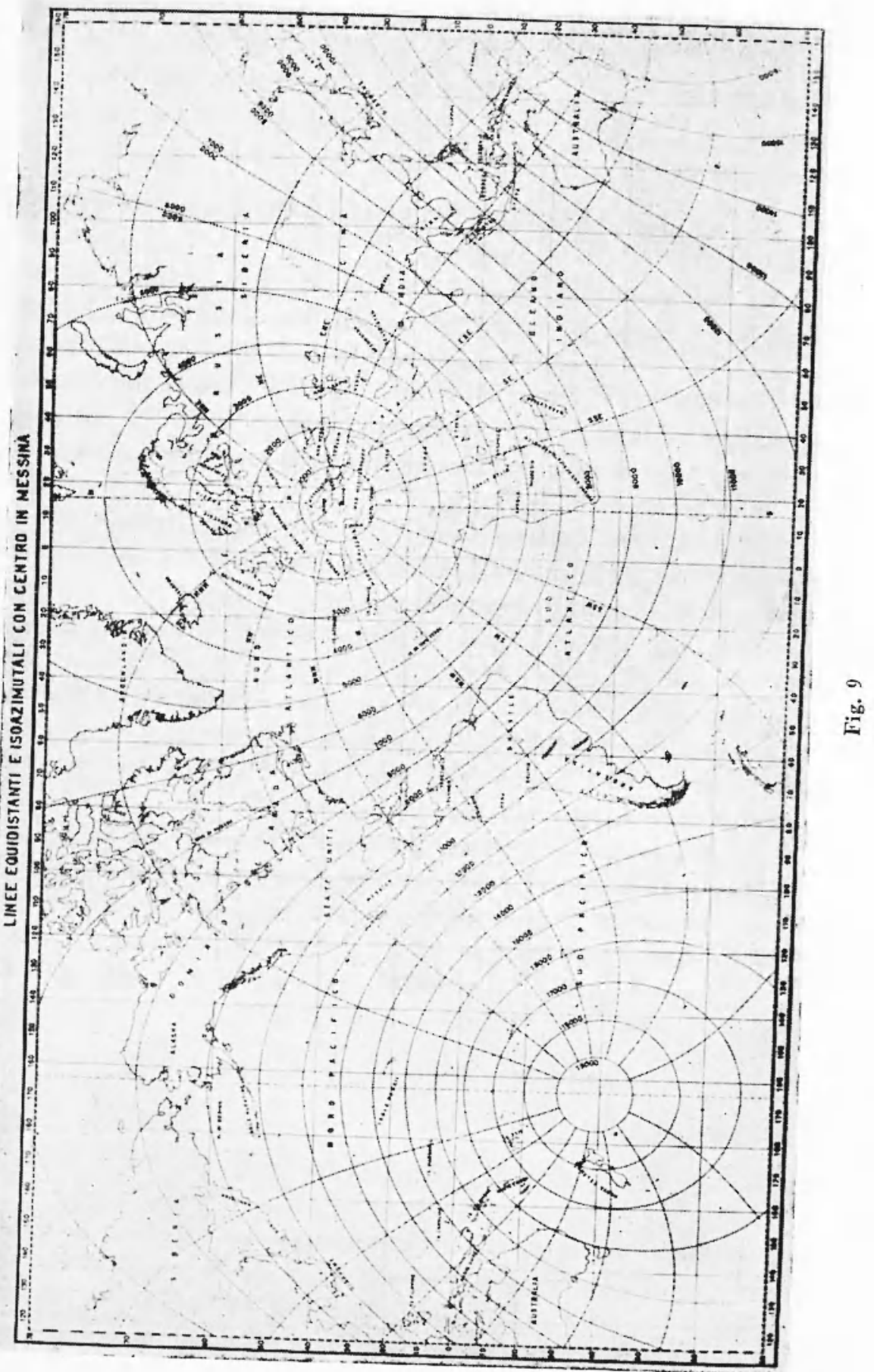




$\begin{array}{lccccccc} & \begin{array}{c}\text { Compo- } \\ \text { nente }\end{array} & \text { T. } & \text { Vo } & \text { Smorz. } & \text { h } & \text { r/T } & \text { D } \\ \text { Wiechert } 200 \mathrm{~K}_{\mathrm{g} .} & \text { NS } & 3 *, 7 & 121,8 & \text { aria } & 0,332 & 0,062 & 30 \\ \text { Wiechert - } & \text { EW } & 3 *, 8 & 110,7 & \text { aria } & 0,270 & 0,059 & 30 \\ \text { Wiechert } 80 \mathrm{Kg} . & \mathrm{V} & 4 \bullet, 3 & 112,5 & \text { aria } & 0,249 & 0,072 & 30\end{array}$

Le corrispondenti curve di ingrandimento dinamico sono riportate nella fig. 6.

La registrazione sistematica ha avuto inizio nell'agosto del 1947, in un primo tempo coi due soli sismografi Wiechert. Nel febbraio 1951 ha avuto inizio il regolare funzionamento dello Sprengnether e, infine, nel dicembre dello stesso anno la stazione ha iniziato la sistematica regristrazione in tutta la sua attuale efficienza. I risultati dello spogrlio delle registrazioni vengono mensilmente pubblicati nel bollettino dell'Istituto Nazionale di Geofisica.

In tutto l'anno 1952 sono stati registrati complessivamente 673 terremoti, di cui: 401 lontanissimi (u), 28 lontani ( $r$ ), 138 vicini (v), 87 locali (d), 19 non caratterizzabili.

Nelle figg. 7, 8 sono riportati alcuni esempi di registrazioni ottenute con i diversi tipi di strumenti.

Nella fig. 9 è riportata una carta di linee equidistanti ed isoazimutali, allestita allo scopo di determinare rapidamente gli epicentri provvisori usufruendo dei dati della sola stazione di Messina, qualora il carattere delle registrazioni ottenute lo consenta.

La posizione della stazione sismica di Messina, prossima a zone fortemente anomale dal punto di vista geofisico - in particolare sismico - la rende di eccezionale interesse per lo studio dei fenomeni sismici e delle caratteristiche fisiche della crosta terrestre.

E quindi griustificato il proposito di farne un caposaldo della rete sismica italiana.

Messina - Osservatorio dellI.N.G. - Dicembre 1952.

\section{RIASSUNTO}

Si danno ampie notizie sulla nuova stazione sismica di Messina, facente parte della rete sismologica dell'Istituto Nazionale di Geofisica.

Gli strumenti a registrazione meccanica (Wiechert 200, Wiechert $80 \mathrm{~kg})$ e quelli " registrazione fotografica (Galitzin e Sprengnether) 
fanno della stazione sismica di Messina uno dei raposaldi della rete sismologica italiana.

\section{SLMMARY}

Complete information is given on the new seismic station of Messina taking part in the seismological net of the Instituto Nazionale di Geofisica.

The mechanical registration instruments (Wiechert 200, Wiechert $80 \mathrm{~kg}$ ) and those for photographic registration (Galitzin and Sprengnether) matie the seismic station of Messina one of the most important of the Italian seismological net. 